\title{
O OLHAR DO PACIENTE SOBRE O CÂNCER INFANTOJUVENIL E SUA PERCEPÇÃO ACERCA DE SEUS SENTIMENTOS E EMOÇÕES DIANTE DO VIDEOGAME ATIVO
}

\author{
PATIENTS' VIEW OF CHILD-YOUTH CANCER AND THEIR PERCEPTION ABOUT \\ THEIR FEELINGS AND EMOTIONS TOWARD ACTIVE VIDEOGAME
}

\begin{abstract}
LA MIRADA DEL PACIENTE SOBRE EL CÁNCER INFANTOJUVENIL Y SU PERCEPCIÓN ACERCA DE SUS SENTIMIENTOS Y EMOCIONES FRENTE AL VIDEOJUEGO ACTIVO
\end{abstract}

\author{
Talita Grazielle Pires de Carvalho*, Ana Raquel Mendes dos Santos*, \\ Maritza Lordsleem Silva*, Ameliane da Conceição Reubens Leonídio*, \\ Priscilla Pinto Costa da Silva ${ }^{* *}$, Iraquitan de Oliveira Caminha ${ }^{* * *}$, \\ Clara Maria Silvestre Monteiro de Freitas*
}

Palavras chave: Hospitalização. Neoplasias. Jogos de vídeo. Emoções.

\begin{abstract}
Resumo: Objetivou-se conhecer e analisar a percepção de crianças e adolescentes hospitalizados em relação ao câncer/tratamento e seus sentimentos e emoções relacionados à prática do videogame ativo no ambiente hospitalar. Trata-se de um estudo qualitativo do tipo pesquisa participante envolvendo 14 pacientes de 7 a 17 anos, internados em um centro hospitalar de onco-hematologia pediátrica. Para coleta utilizaram-se entrevista semiestruturada, observação participante e diário de campo. Nas intervenções utilizou-se o X-Box 360 com Kinect, com jogos de esporte e dança. Os dados foram analisados através da Análise de Conteúdo. Os entrevistados demostraram ter conhecimento sobre a doença e o tratamento. $O$ videogame ativo foi apontado como uma possível estratégia para romper com a tristeza e o tédio, por proporcionar interação social e uma vivência de sentimentos e emoções positivas no ambiente hospitalar.
\end{abstract}

Keywords: Hospitalization. Neoplasms. Video games. Emotions.

Palabras clave: Hospitalización. Neoplasias. Videojuegos. Emociones.

\begin{abstract}
This study aimed at knowing and analyzing knowledge of children and adolescents admitted in hospitals about cancer/treatment and their feelings and emotions linked to the practice of active videogame in the hospital environment. It is a qualitative research-type study involving fourteen patients aged 7-17, admitted to an onco-hematology pediatric hospital center. The study applied semi-structured interviews, participant observation and field diary to obtain data. Interventions used the X-Box 360 with Kinect sports and dance games. Data were subjected to content analysis. The subjects demonstrated they knew about cancer disease and treatment. Patients pointed out active video game as a possible strategy to counter sadness and boredom because the games provided social interaction and positive feelings and emotions in the hospital environment.
\end{abstract}

Resumen: Se objetivó conocer y analizar la percepción de niños y adolescentes hospitalizados en relación al cáncer/tratamiento y a sus sentimientos y emociones relacionados a la práctica del videojuego activo en el ambiente hospitalario. Se trata de un estudio cualitativo del tipo investigación participante que involucra a 14 pacientes de 7 a 17 años, internados en un centro hospitalario de oncohematología pediátrica. Para la recolección de datos se utilizó entrevista semiestructurada, observación participante y diario de campo. En las intervenciones se utilizó el X-Box 360 con Kinect, con juegos de deporte y danza. Los datos fueron analizados a través del análisis de contenido. Los entrevistados demostraron tener conocimiento sobre la enfermedad y el tratamiento. El videojuego activo fue señalado como una posible estrategia para romper con la tristeza y el aburrimiento, por proporcionar interacción social y una vivencia de sentimientos y emociones positivas en el ambiente hospitalario.
*Universidade de Pernambuco. Recife, PE, Brasil.

E-mail: talitagraziellepires@gmail.com; raquel_mdss@ hotmail.com; maritzalordsleem @ hotmail.com; ameliane.reubens@yahoo.com.br; clarasilvestre@uol.com.br

**Universidade Federal do Rio Grande do Norte. Natal, RN, Brasil. E-mail: laprisci@gmail.com

***Universidade Federal da Paraíba. João Pessoa, PB, Brasil. E-mail: caminhairaquitan@gmail.com

Recebido em: 17-04-2017 Aprovado em: 10-06-2017

DOI: http://dx.doi.org/10.22456/1982-8918.72695 (c) (1) (8) Licence 


\section{INTRODUÇÃO}

O câncer corresponde a um conjunto de doenças que têm em comum a proliferação de células anormais, podendo ocorrer em qualquer local do organismo, sendo 0 câncer infantojuvenil (crianças e adolescentes entre zero e 19 anos) um conjunto que apresenta características próprias, principalmente com relação à histopatologia e ao comportamento clínico (INSTITUTO NACIONAL DE CÂNCER, 2015; 2016).

Nas últimas décadas, o progresso do tratamento oncológico na infância e na adolescência foi bastante significativo, refletindo numa maior possibilidade de cura para pacientes quando diagnosticados precocemente e tratados em centros especializados, além da melhora na qualidade de vida após o tratamento adequado (INSTITUTO NACIONAL DE CÂNCER, 2008).

Tão importante quanto o tratamento do câncer em si é a atenção dada aos aspectos sociais da doença, uma vez que a criança e 0 adolescente doente devem receber atenção integral, no seu contexto familiar. A cura não deve se basear somente na recuperação biológica, mas também no bem-estar e na qualidade de vida do paciente (INSTITUTO NACIONAL DE CÂNCER, 2015).

Nos últimos tempos, tem havido uma preocupação crescente com os efeitos do ambiente hospitalar sobre o desenvolvimento infantil (DIAS et al., 2013; HOSTERT; ENUMO; MOTTA LOSS, 2014), sendo inegável a vulnerabilidade de crianças e adolescentes quando hospitalizadas. Neste ambiente, o paciente encontra-se afastado do seu locus familiar, dos amigos e da escola para submeter seu corpo a processos dolorosos e desagradáveis, tornando-o vulnerável a sentimentos e emoções nocivos a sua saúde (MITRE; GOMES, 2004; MALTA; SCHALL, 2012; CATAUDELLA; ZELCER, 2012).

Para Le Breton (2009, p. 117), os sentimentos e as emoções ligam-se "[...] à interpretação que 0 indivíduo faz do acontecimento que 0 afeta moralmente, modificando sua relação com o mundo [...] seja por anos, seja por alguns segundos". Um ambiente novo, com pessoas desconhecidas, e a limitação das atividades em geral tendem a se configurar como uma situação difícil de ser enfrentada. Portanto, a vivência no ambiente hospitalar pode se tornar estressante, com impacto sobre o estado emocional e psicológico do paciente, desencadeando sentimentos e emoções negativos (PAULA; FOLTRAN, 2007).

Tal vivência pode ser desvantajosa para recuperação da saúde por ocasionar sofrimento, irritabilidade, regressão, baixa imunidade e dificuldades no desenvolvimento, muitas vezes associados às condições físicas inadequadas, bem como procedimentos hospitalares que causam desconforto e profissionais de saúde com relacionamento distante (FERREIRA et al. 2014). Esses fatores afetam o estado emocional, podendo produzir mudanças no sistema imunológico, provocando alterações nas respostas ao tratamento (BROD et al., 2014). Assim, a vivência de sentimentos e emoções negativas podem desencadear uma resposta negativa ao sistema imunológico, repercutindo no tratamento.

Para amenizar tal situação, foi criada a lei oํ 11.104, de 21 de março de 2005 (BRASIL, 2005), que obriga hospitais que oferecem atendimento pediátrico em regime de internação a contar com brinquedotecas nas suas dependências. Esse espaço deve ser provido de jogos e brinquedos educativos destinados a estimular a interação lúdica entre pacientes e 
acompanhantes, contribuindo para a construção e fortalecimento das relações afetivas entre paciente e meio social.

Por meio da brincadeira, crianças e adolescentes são capazes de alterar o ambiente hospitalar, refletindo de maneira positiva na forma como eles passam a se ver e entender a hospitalização, auxiliando no crescimento, no desenvolvimento psicomotor e na promoção do bem-estar e da saúde (OLIVEIRA et al., 2009; CARVALHO; BEGNIS, 2006; COSTA JR; COUTINHO; FERREIRA, 2006; VAZ; VIEIRA; GONÇALVES, 2005, FERREIRA et al. 2014).

A saúde aqui é entendida como: "[...] ter meios de traçar um caminho pessoal e original, em direção ao bem-estar físico, psíquico e social" (DEJOURS, 1986, p.11), sendo um estado do qual procuramos nos aproximar. Dejours (1986) afirma que a saúde não é um estado de estabilidade, a saúde é algo que muda o tempo todo, caraterizada como uma sucessão de compromissos com a realidade. Logo, ser saudável é sempre permitir-se ter esperança.

Com base na perspectiva do brincar (HUIZINGA, 2014), torna-se importante considerar o brinquedo como uso terapêutico na busca pela saúde, podendo ser considerado um fator de proteção para o equilíbrio emocional e psicológico dos pacientes pediátricos. Nesse sentido, o estudo de Ferreira et al. (2014) destaca que o lúdico no hospital possibilita maior aceitação da situação vivida, melhor adaptação e familiarização ao ambiente hospitalar, proporcionando a continuidade das atividades dos pacientes e influenciando na sua recuperação durante a hospitalização.

Uma estratégia que vem sendo utilizada como recurso lúdico terapêutico são os jogos do videogame ativo. Conforme Lima e Gomes (2014), esses jogos são capazes de mudar comportamentos, educar e formar os jogadores. Os autores fizeram um levantamento de 14 jogos com foco em processos de reabilitação de algumas doenças crônicas como: asma, obesidade, AIDS, diabetes, depressão e câncer, bem como sua utilização como ferramenta para melhoria da precisão motora, focando a fisioterapia e atividade física, confirmando a importante potencialidade dos jogos para promover benefícios físicos e psicológicos em sujeitos com doenças crônicas.

Nessa perspectiva, a prática do videogame ativo, ao se apresentar como uma atividade lúdica, surge como uma possibilidade de expressão de emoções, preferências, receios e hábitos; mediação entre o mundo familiar e situações novas ou ameaçadoras, que permitem elaborar experiências desconhecidas ou desagradáveis. 0 videogame ativo é algo prazeroso, capaz de trazer alegria e resgatar a condição de "ser criança/adolescente" que por vezes é perdida dentro do contexto de hospitalização (LIMA; GOMES, 2014; OLIVEIRA; MITRE; GOMES, 2004; DIAS; ROAZZI, 2003). Nesse sentido, objetivou-se conhecer e analisar a percepção de crianças e adolescentes hospitalizados em relação ao câncer/tratamento, considerando seus sentimentos e emoções relacionados à prática do videogame ativo no ambiente hospitalar.

\section{TRAJETÓRIA METODOLÓGICA}

Trata-se de um estudo qualitativo do tipo pesquisa participante, caracterizada pela interação entre pesquisadores e membros da situação investigada (GIL, 2002). Sendo, portanto, uma atividade educativa de investigação e ação social, exigindo a implicação do pesquisador e dos sujeitos envolvidos na pesquisa (BRANDÃO, 1981). 
O estudo teve como cenário a brinquedoteca do Centro Hospitalar de Oncohematologia Pediátrica (CEONHPE) do Hospital Universitário Oswaldo Cruz (HUOC), referência em tratamento de câncer, localizado em Recife, Pernambuco. Os dados foram coletados entre maio e junho de 2015. Foram realizadas de quatro a seis sessões por dia nas terças e quintas, durante o período da tarde das 14 às 17 horas. No total foram realizados dez dias de intervenção.

Vinte pacientes foram autorizados pela equipe médica a participar do estudo. No entanto, seis foram excluídos da amostra, dos quais três desistiram de jogar alegando cansaço, preferindo permanecer em seus leitos; dois participaram do jogo, mas não realizaram a entrevista; e um paciente participou do jogo e realizou a entrevista, porém tinha idade superior a 19 anos, estando fora da faixa etária estabelecida como critério de inclusão. Por fim, o presente estudo contou com a participação de 14 pacientes com idade entre sete e 17 anos.

Para as sessões de videogame ativo, foi escolhido o console $X$-box 360 com Kinect a fim de facilitar os movimentos corporais dos pacientes, pois este captura a imagem do corpo fazendo uma leitura do movimento em 3D. O jogador não necessita estar ligado a um dispositivo, e o corpo do usuário se torna o agente controlador do jogo. Dentre os jogos selecionados estão - Game Kinect Sports - Xbox 360 (Ultimate Collection), que reúne 13 jogos de esporte, os quais foram selecionados levando em consideração as condições físicas dos pacientes para a prática do futebol, golfe, esqui, tênis, arremesso de dardos, boliche e tênis de mesa; 0 Just Dance Kids 2014 - Xbox 360 Kinect (Ubisoft) e o Just Dance Disney Party - Xbox 360 Kinect (Ubisoft), que reúnem coreografias e músicas direcionadas ao público infantil e adolescente.

Buscando a qualidade da pesquisa e uma compreensão aprofundada do fenômeno estudado, a coleta e a análise dos dados se deram por meio da triangulação dos dados de três instrumentos de coleta (FLICK, 2009), sendo eles: 1. A entrevista semiestruturada com o paciente, por ser uma técnica eficiente para obtenção de dados em profundidade acerca do fenômeno estudado, podendo ser aplicada com pessoas que não sabem ler e escrever, permitindo um contato mais próximo do participante da pesquisa e possibilitando identificar dúvidas e estabelecer uma relação de confiança necessária na pesquisa com os mais jovens (OLIVEIRA et al., 2010); 2. O roteiro de observação participante, o qual direcionou as observações do profissional de Educação Física durante as sessões do videogame ativo; e 3. O diário de campo, que além de possibilitar o registro minucioso do dia a dia da pesquisa, possibilitou uma reflexão da ação profissional desenvolvida, revendo seus limites e desafios, auxiliando no aprofundamento do conhecimento do fenômeno estudado. Para caracterização dos participantes foram utilizados o questionário sociodemográfico (respondido por um adulto responsável pelo paciente) e o Protocolo de consulta ao prontuário do paciente.

A princípio, foram realizadas uma visita e uma reunião de sondagem junto à médica responsável pelo CEONHPE-HUOC, sendo identificada a necessidade de jogos e brincadeiras ativas para os pacientes em período de internação. Em seguida ocorreram quatro visitas à brinquedoteca para observar e conversar com os pacientes. Posteriormente, oito visitas foram realizadas para identificar, junto com os responsáveis da equipe médica, de enfermagem e do Grupo de Apoio à Criança Carente com Câncer de Pernambuco (GAC-PE) responsável pelo centro, se haveria condições de submeter os pacientes internados na enfermaria às sessões de jogos com o videogame ativo. A partir daí desenvolveu-se um trabalho em conjunto com a equipe médica, que avaliava e autorizava diariamente os pacientes aptos para o jogo, a 
equipe do GAC-PE que ficou responsável pelo suporte diário durante as sessões e a equipe de profissionais de Educação Física que coordenou e acompanhou o trabalho referente aos jogos.

Após o consentimento da equipe, foram iniciadas as sessões do videogame ativo, de forma individual ou em dupla, com duração de 30 minutos por sessão, nas quais os pacientes escolhiam os jogos que mais Ihes agradassem, dentre as opções oferecidas. Ao final da primeira sessão de jogo de cada paciente, a entrevista era realizada pelo profissional de Educação Física, adotando os critérios sugeridos por Flick (2009), iniciando uma breve introdução sobre o procedimento e o tema abordado, com perguntas acessíveis cuidando para não influenciar os entrevistados com opiniões particulares ou tendenciosas. As entrevistas foram registradas com um aparelho gravador de voz sendo posteriormente arquivadas e transcritas. A observação participante e o diário de campo foram preenchidos pelo profissional de Educação Física durante ou ao final das sessões.

Para a análise dos dados obtidos das transcrições das entrevistas, da observação participante e do diário de campo utilizou-se a Análise de Conteúdo de Bardin (2011), por meio dos seguintes passos: 1. Construção do corpus de análise a partir da transcrição do material coletado; 2. Leitura flutuante; 3. Definição e identificação das unidades de registros no texto; 4. Nomeação e identificação das unidades de significação; 5. Quantificação das unidades de significação em cada corpus; 6 . Agrupamento dos temas para construção das categorias; 7. Nomeação, quantificação e descrição das categorias; e 8. Associação das categorias às características dos entrevistados e ao conhecimento científico existente (BARDIN, 2011). Para a análise dos dados foram definidas quatro categorias, das quais duas serão tratadas neste estudo e apresentadas com auxílio do software GoDiagram Express versão 2.6.2.

A pesquisa foi aprovada pelo Comitê de Ética em Pesquisa com o CAAE 42625315.5.0000.5207 e parecer 1.013.574. A fim de respeitar as questões éticas da Resolução 466/12 do Conselho Nacional de Saúde/MS, que regulamenta as diretrizes e normas de pesquisa envolvendo seres humanos, os pacientes que aceitaram participar como voluntários tiveram o Termo de Consentimento Livre e Esclarecido e o Termo de Assentimento devidamente assinados por seus responsáveis legais por serem menores de 18 anos.

\section{RESULTADOS E DISCUSSÃO}

Foram entrevistados 14 pacientes, sendo quatro meninas e dez meninos, com idades entre sete e 17 anos, sendo sete diagnosticados com leucemia linfoide aguda, quatro com osteossarcoma, um com leucemia mieloide aguda, um com linfomas de grandes células e um com neuroblastoma. Dois dos participantes apresentaram amputação em um dos membros inferiores. Todos os pacientes estavam internados há mais de cinco dias. No tocante à renda familiar, oito dos responsáveis pelos pacientes declararam ter uma renda mensal de até um salário mínimo e seis declararam uma renda de até 4,5 salários mínimos. Dentre os pacientes, nove residiam no estado de Pernambuco, dois em Alagoas, um na Bahia, um na Paraíba e um em Sergipe, dos quais dez residiam em cidades do interior.

Buscou-se conhecer a percepção de crianças e adolescentes sobre o câncer, 0 tratamento e a prática do videogame ativo durante o período de hospitalização. Nesse sentido, emergiram duas categorias: 1. "Conhecimento sobre o câncer e seu tratamento", em que foi considerada a percepção do paciente em relação à sua doença e tratamento; e 2. "Sentimentos 
e emoções relacionados à sessão de videogame ativo", que apresenta a percepção dos pacientes em relação aos seus sentimentos e emoções diante da prática do videogame ativo, durante o período de hospitalização.

Com o intuito de diferenciar os discursos, os pacientes serão identificados por meio da letra "P", seguida pelo gênero representado através da letra "F" para feminino ou "M" para masculino, o número e a idade do paciente no dia da entrevista.

\subsection{A percepção de crianças e adolescentes com câncer sobre a doença e seu tratamento}

O processo de hospitalização, especialmente infantil, deve proporcionar o mínimo de situações traumáticas para o paciente. Para isso, faz-se necessário, principalmente na faixa etária escolar, que os participantes sejam informados quanto ao motivo da internação, os procedimentos realizados e as rotinas hospitalares (DIAS et al., 2013).

No diagrama abaixo (Figura 1), está identificada a categoria "Conhecimento sobre 0 câncer e seu tratamento" apresentada na cor cinza escuro. Abordou-se o conhecimento dos pacientes, participantes do estudo, a respeito da doença. A partir dessa categoria, derivaram três subcategorias, indicadas na cor cinza claro, sendo elas: "Conhecimento sobre o motivo da internação", "Conhecimento sobre a doença" e "Sentimentos relacionados ao tratamento e a doença". Cada subcategoria apresenta algumas variáveis que estão identificadas na cor branca.

Figura 1 - Conhecimento sobre o motivo da internação, o câncer, seu tratamento e sentimentos percebidos pelos pacientes.

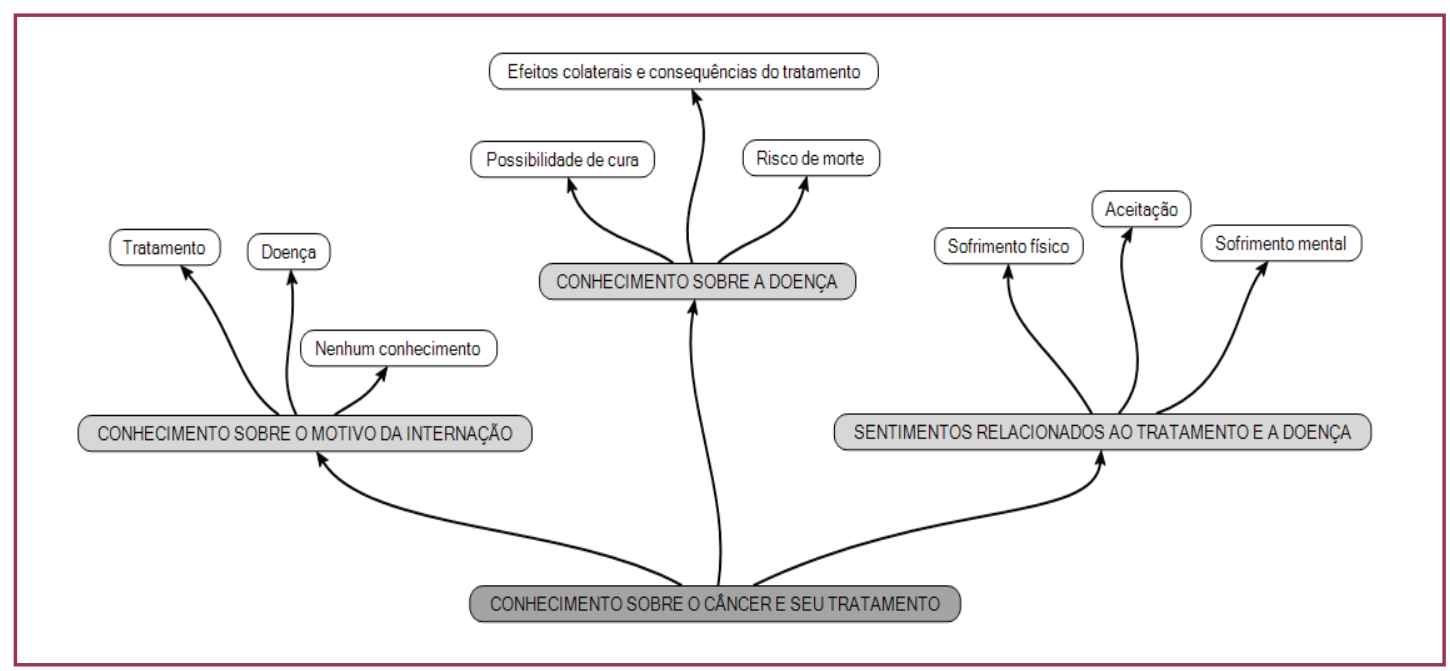

Fonte: Dados da pesquisa

Os discursos apontam que 13 participantes detêm certo conhecimento sobre o motivo da sua hospitalização, como fica destacado no depoimento do paciente 7: "Eu vim pra cá pra tomar QT" (quimioterapia) (P.F. 7-14 anos). No que se refere à doença, ao tratamento, às suas consequências e efeitos colaterais, do risco de morte, de amputação, o relato do paciente 11 revela: "É uma doença que pode matar e eu também posso correr o risco de perder a perna" (P.M. 11-11 anos). Sobre a possibilidade de cura, fica ressaltado na fala dos pacientes: "[...] Que ela é uma doença que é fácil de curar, mas também pode matar, e que caso não se cure 
com a quimioterapia tem transplante" (P.M. 6-16 anos), "[...] Que pode curar, que pode matar" (P.F. 14-8 anos).

Esse contexto se apresenta como resultado do trabalho realizado pelas equipes de saúde do hospital e do GAC, no sentido de investir em estratégias de interação comunicativa médico-cuidador-paciente, em que a participação do paciente em seu tratamento ganha um espaço importante. Para Kohlsdorf e Seidl, (2011), a criança deve participar efetivamente do processo terapêutico e das negociações sobre o tratamento, cabendo ao médico e aos pais, como participantes dominantes da interação comunicativa, possibilitar um ambiente promotor do desenvolvimento da criança, visando menores custos comportamentais associados ao tratamento, tanto para profissionais de saúde quanto para cuidadores e pacientes.

Os pacientes demonstram boa aceitação do tratamento apesar das dificuldades e sofrimento físico como enjoos, fraqueza e cansaço, como apontam os relatos: "[...] Demorado (leve sorriso) ... cansativo!" (P.M. 1-13 anos); "[...] Enjoada... Ah! Não! Na verdade eu fiquei feliz porque descobriu o que eu tinha, porque eu só sentia dor e não descobria o que era! Aí quando descobriu foi bem tranquilo" (P.F. 8-17 anos). E do sofrimento mental, como a saudade de casa e da família, o desânimo e a tristeza, como aponta o relato da Paciente 7 "[...] Fraca, desanimada." (P.F. 7-14 anos).

Treze participantes demonstraram enxergar o tratamento como um "mal necessário", como está destacado nos depoimentos dos Pacientes 2 e 11: "No começo eu me senti triste, lógico, mas depois é superar e tratar pra ficar bom... É bem chato, mas é necessário pra ficar melhor, então o jeito é fazer, né!" (P.M. 2-14 anos); "Bem, no início eu num gostava muito não, que eu num ficava em casa, mas eu sei que é pra o meu bem, então é melhor aceitar. Quanto mais eu aceitar melhor!" (P.M. 11-11 anos). Apenas uma paciente, durante as entrevistas, expressou não ter conhecimento sobre a doença e o motivo da sua internação, como é mostrado na conversa entre a profissional de Educação Física (PEF) e a Paciente 9 , de 7 anos:

\author{
PEF: 'O que você sabe sobre sua doença?' \\ Paciente 9: 'Não sei!' \\ PEF: 'Por que você não sabe?' \\ Paciente 9: 'Ninguém disse pra mim!' \\ PEF: 'Como você está se sentindo no hospital, agora?' \\ Paciente 9: 'Cansada! Com saudade da minha cidade, da minha família!' (Cabeça \\ baixa).
}

Esta situação justifica-se pelo fato de a paciente estar no início do tratamento de leucemia linfoide aguda, durante a primeira sessão de quimioterapia, e está apresentando sintomas depressivos, de acordo com o registro no prontuário do paciente e durante reunião entre a PEF e a equipe médica do CEONHPE- HUOC, registrada no diário de campo.

Quando comparadas a outras doenças prevalentes na população infantil, as neoplasias apresentam dificuldades comportamentais intensas, em razão do tratamento agressivo e extenso, dos efeitos colaterais da medicação, da repetição de procedimentos invasivos, das alterações na rotina e dinâmica familiar e das incertezas em relação ao sucesso do tratamento (MALTA; SCHALL, 2012; CATAUDELLA; ZELCER, 2012; KOHLSDORF; SEIDL, 2011). $O$ diagnóstico e 0 tratamento de câncer incluem: uma etiologia multifatorial e relativamente desconhecida, a complexidade dos protocolos terapêuticos e as representações sociais 
associadas às neoplasias, como a vivência de perdas, sofrimento e morte, aspectos que contribuem para o aumento da aversão ao tratamento, dificultando a comunicação entre equipe de saúde, cuidador e paciente (KOHLSDORF; SEIDL, 2011).

As informações sobre o câncer são, em geral, muito complexas e permeadas pela carga emocional e incerteza, o que propicia condições para uma comunicação ineficiente e para consequências prejudiciais à vivência do tratamento, como manifestações de ansiedade, adesão insatisfatória às recomendações terapêuticas, sobrecarga de cuidados e baixa retenção de informações. Nesse contexto, Kohlsdorf e Seidl (2011) destacam que a qualidade da interação comunicativa pode influenciar o impacto do diagnóstico e tratamento do câncer na infância, pois quanto maior a interação da criança na comunicação, melhor será sua adaptação e autonomia em relação a elementos aversivos do tratamento, minimizando as dificuldades inerentes ao processo de adoecimento.

Para Borges, Nascimento e Silva (2008), a utilização da interação comunicativa associada às estratégias para minimizar os efeitos negativos oriundos da hospitalização potencializa os ganhos relacionados à aprendizagem e ao repertório comportamental de pacientes pediátricos, fazendo-se necessária durante esse período. Neste sentido, as atividades lúdicas com jogos e brincadeiras aparecem como catalisadores no processo de recuperação e adaptação da criança hospitalizada. Isso porque tais atividades viabilizam a construção de uma realidade própria e singular, possibilitando a expressão da criatividade, de sentimentos e emoções (HUIZINGA, 2014; GOMES-DA-SILVA, 2011).

\subsection{Sentimentos e emoções de pacientes em relação ao videogame ativo no ambiente hospitalar}

Da categoria "Sentimentos e emoções relacionados à sessão de videogame ativo", ilustrada na Figura 2 na cor cinza escuro, emergem três subcategorias, indicadas na cor cinza claro, sendo elas: "Sentimentos e emoções antes de jogar o videogame ativo", "Sentimentos e emoções durante o jogo de videogame ativo" e "Sentimentos e emoções após jogar 0 videogame ativo". Cada subcategoria mencionada apresenta algumas variáveis que estão identificadas na cor branca.

Figura 2 - Sentimentos e emoções antes, durante a após a sessão de videogame ativo.

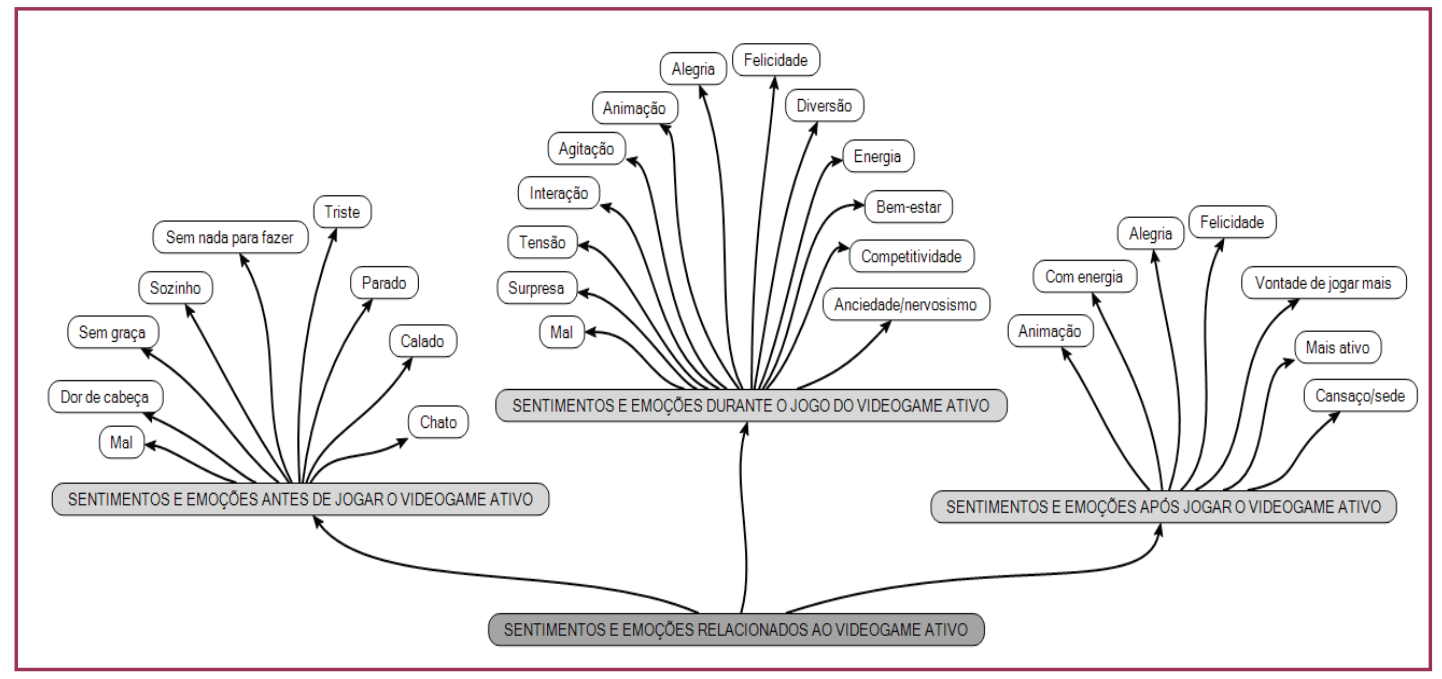


Os discursos de crianças e adolescentes participantes do estudo apontaram que estes pacientes se sentiam tristes e entediados antes da sessão do videogame ativo, como pode ser observado nos relatos dos pacientes quando questionados acerca de como eles estavam se sentindo antes de jogar o videogame ativo: "Sonolenta, de saco cheio de tá aqui!" (P.F. 8-17 anos), "Eu tava triste sem brincar, aí foi, chegou isso (X-box) e eu me animei! (Sorriso)" (P. M. 10-7 anos); "Mal! Num tinha nada pra fazer na brinquedoteca, só tinha boneca!" (P.F.14-8 anos).

Segundo Oatley e Jenkins (2002), na tristeza há uma reorientação interna, o que direciona a concentração para o próprio sujeito que reavalia a si próprio em relação ao que aconteceu, havendo uma resignação acerca do evento que a causou. Para os autores, a tristeza está relacionada com o desgosto e a depressão, podendo ser considerada patológica quando prolongada. Para Brod et al. (2014), os estados emocionais negativos podem desencadear estresse agudo e doenças crônicas, em decorrência da resposta de células imunológicas de proteção em uma ampla variedade de doenças relacionadas ao sistema imunológico, incluindo o câncer, além de refletir de forma negativa na recuperação do paciente durante o tratamento, pois, quando sensações de estresse ou raiva se tornam persistentes, a nossa resposta imune pode tornar-se notavelmente diminuída ou desequilibrada.

Ao ter conhecimento de que poderiam jogar o videogame ativo no hospital, alguns pacientes se animaram, expressando alegria e interesse no jogo, relatando que ajudaria a passar o tempo de maneira prazerosa, divertida e mais ativa. Observa-se que ao questionar os pacientes a respeito de como se sentiram durante o jogo, os sentimentos mais descritos nas entrevistas foram felicidade, sentir-se bem, alegria e diversão, como mostram os depoimentos: "Muito melhor! [...] felicidade, emoção, tensão, muitas coisas!" (P.M. 1-13 anos) e "Me senti bem, porque é uma coisa prazerosa de se fazer, né! Jogar aqui que nós gostamos... Eu diria só alegria e felicidade mesmo! Só alegria e felicidade!" (P.M. 2- 4 anos).

Quando se está feliz o cérebro torna a organização cognitiva mais flexível, produzindo associações pouco habituais. Para Oatley e Jenkins, (2002, p.308-309):

\begin{abstract}
[...] A felicidade estimula as pessoas a tentarem alcançar objetivos mais elevados, bem como a continuar o que estão a fazer e a resistir a mudança para outro estado. Quando estamos felizes somos igualmente mais úteis e cooperativos. Algumas atividades são estabelecidas meramente para gozar - os jogos, as férias, a leitura de romances, as conversas. Não se trata de que durante tais estejam ausentes; antes envolvemo-nos de forma feliz no que estamos a fazer, sem distrações, e somos capazes de lidar facilmente com o que acontece à medida que ocorre.
\end{abstract}

Tal fato reflete de maneira benéfica no tratamento do câncer. Para Brod et al. (2014), os estados emocionais positivos ou bem-estar emocional têm um efeito positivo no sistema imunológico, ajudando na reabilitação do paciente. Desta forma, percebe-se que o sistema imunológico pode ser influenciado pelo ambiente social.

Outro ponto importante destacado durante as entrevistas foi a questão da socialização promovida pelo jogo, como observado no diálogo entre o PEF e o Paciente 6, de 16 anos, hospitalizado há 4 meses:

PEF: 'Como você se sentiu durante o jogo do videogame ativo?'

Paciente 6: 'Ah, eu fico muito feliz porque tem os meninos pra interagir, tem as enfermeiras vendo jogar, tem os meus colegas que eu vejo, e os que eu não 
vejo, que eu fico em cima e eles ficam em baixo (enfermarias do $5^{\circ}$ e $4^{\circ}$ andar). $E$ quando eu desço pra cá eu vejo todo mundo.'

PEF: 'E o que você sentiu no momento em que estava jogando?’

Paciente 6: 'Emoção!'

PEF: 'Descreva essa emoção.'

Paciente 6: 'Descrever, como eu posso descrever essa emoção? Fico muito feliz, o coração acelera é muito engraçado!'

Esse diálogo aponta para a necessidade de interação social e criação de vínculos de amizade entre indivíduos com os quais os pacientes possam se identificar, sendo a interação social de grande importância para o desenvolvimento destes pacientes. Para Taille, Oliveira e Dantas (1992, p.11), "[...] o homem é um ser essencialmente social, impossível, portanto, de ser pensado fora do contexto da sociedade em que nasce e vive".

A situação de doença/hospitalização tende a diminuir a interação do paciente com familiares e amigos, se opondo ao aumento na necessidade de segurança e orientação, oriundas da relação com esses grupos, uma vez que durante a fase da adolescência cresce a necessidade de ligação com grupos de identificação, a fim de desenvolver autonomia, independência e autoafirmação (MENOSSI; LIMA, 2000).

Após a sessão de videogame ativo, sete dos pacientes participantes da pesquisa expressaram o desejo de continuar jogando e poder jogar outras vezes, como relata a Paciente 7: "Ah! Tô me sentindo animada! Mas um pouquinho triste porque parei de jogar." (P.F. 7-14 anos); o Paciente 11: "Bem, eu tô sentindo que eu queria jogar de novo, mas eu vou ter que esperar até quinta!" (P.M. 11-11 anos) e o Paciente 12: "Tava se sentindo bem... com vontade de brincar muito!" (P.M. 12-7 anos). Os pacientes também relataram gostar de observar os outros pacientes jogando, como visto no relato do Paciente 4: "Bem! Tô me sentindo bem! Com vontade de olhar os colegas jogando!" (P.F. 4-13 anos), sendo esta uma forma encontrada para continuar participando do jogo e interagindo com os demais pacientes.

Embora a aceitação do videogame ativo seja mais prevalente, uma paciente relatou não ter se sentido bem após o jogo, indo para a enfermaria a fim de ser avaliada pelo médico. Foi informado que a paciente estava intoxicada, pois seu organismo não teria eliminado a medicação de forma eficiente, desencadeando sintomas como "dor de cabeça e cansaço" (P.F. 9-7 anos) descritos na entrevista.

Durante as observações foi constatado que, no início das sessões do videogame ativo, a curiosidade e ansiedade para jogar, mesmo com o receio devido ao acesso venoso periférico em um dos braços ou a implantação do acesso venoso central, ficaram evidentes entre os pacientes. As limitações dadas pelo uso do acesso venoso foram superadas de diferentes maneiras pelos próprios pacientes, seja imobilizando o braço do lado onde estava implantado 0 acesso venoso central ou segurando o equipo ${ }^{1}$ levemente com a mão para imobilizá-lo. Outra dificuldade enfrentada foi a presença de amputação de membro inferior apresentada por alguns pacientes, o que foi vencido logo na escolha dos jogos, em que o jogo mais escolhido por esse grupo de pacientes foi o tênis (de quadra e de mesa). Os pacientes criaram estratégias que possibilitaram o jogo de forma muito divertida, jogando sem as muletas, ou jogando sentados 
em cadeira de rodas ou em cadeiras comuns, o que não diminuiu a empolgação, a diversão e a competição com os demais colegas que também jogavam.

Os achados apontam que apesar da tristeza oriunda da rotina hospitalar, a estratégia lúdica do videogame ativo se mostrou favorável à promoção da interação social dos pacientes, promovendo a vivência de sentimentos e emoções positivas mesmo durante situações de dificuldades ou sofrimento.

\section{CONSIDERAÇÕES FINAIS}

Apesar da maioria dos pacientes entrevistados ter demonstrado conhecimento sobre 0 câncer e suas possíveis consequências, aceitando, dessa forma, o tratamento como "um mal necessário", os pacientes demostraram sentimentos de tristeza e tédio decorrentes da rotina hospitalar.

Contudo, os resultados encontrados apontaram o videogameativo como uma estratégia eficaz para romper com os sentimentos de tristeza e tédio procedentes da hospitalização, pois esses jogos se mostraram capazes de proporcionar a interação social e a vivência de sentimentos e emoções positivos, como: bom humor, alegria e diversão dentro do ambiente hospitalar, em meio à situação de dificuldade e sofrimento decorrentes do tratamento oncológico. Desta forma, os achados sugerem o videogame ativo como uma possível estratégia para auxiliar no processo de adaptação e enfrentamento do período de hospitalização.

Em razão do tempo de intervenção ter se configurado como uma limitação nesse estudo, sugere-se a realização de novas investigações sobre a temática devido à carência de estudos dessa natureza ligados à temática de estudos socioculturais da Educação Física. As ações desenvolvidas na pesquisa beneficiaram pacientes, facilitando o trabalho da equipe de saúde, contribuindo com a comunidade científica da Educação Física no que se refere ao uso do movimento humano no enfrentamento e tratamento do câncer infantojuvenil.

\section{REFERÊNCIAS}

BARDIN, Laurence. Analise de conteúdo. Lisboa, Portugal: Edições 70, 2011.

BORGES, Emnielle Pinto; NASCIMENTO, Maria do Soares Brandão; SILVA, Silvana Maria Moura. Benefícios das atividades lúdicas na recuperação de crianças com câncer. Boletim Academia Paulista de Psicologia, v. 28, n. 8, n. 2, p. 211-221, 2008.

BRANDÃO, Carlos Rodrigues. Pesquisa Participante. São Paulo: Brasiliense. 1981.

BRASIL. Lei no 11.104, de 21 de março de 2005. Dispõe sobre a obrigatoriedade de instalação de brinquedotecas nas unidades de saúde que ofereçam atendimento pediátrico em regime de internação. Diário Oficial da União, Brasília, DF, 21 mar. 2005. Disponível em: <http://www. planalto.gov.br/ccivil 03/ Ato2004-2006/2005/Lei/L11104.htm>. Acesso em: 4 fev. 2015.

BROD, Samuel et al. 'As above, so below' examining the interplay between emotion and the immune system. Immunology, v. 143, n.3, p. 311-318, 2014. 
CARVALHO, Alysson Massote; BEGNIS, Juliana Giosa. Brincar em unidades de atendimento pediátrico: aplicações e perspectivas. Psicologia em estudo, v. 11, n. 1, p. 109-117, 2006.

CATAUDELLA, Danielle; ZELCER, Shayna. Psychological experiences of children with brain tumors at end of life: parental perspectives. Journal of Palliative Medicine, v. 15, n. 11, p. 1191-1197, 2012.

COSTA JR., Áderson Luiz; COUTINHO, Sílvia Maria Gonçalves; FERREIRA, Rejane Soares. Recreação planejada em sala de espera de uma unidade pediátrica: efeitos comportamentais. Paidéia, v.16, n.33, p. 111-118, 2006.

DEJOURS, Christophe. Por um novo conceito de saúde. Revista Brasileira de Saúde Ocupacional, v. 14, n. 54, p.7-11, 1986.

DIAS, Jucielma de Jesus et al. A experiência de crianças com câncer no processo de hospitalização e no brincar. REME, v.17, n.3, p. 608-613, 2013.

FERREIRA, Naidhia Alves Soares et al. Representação social do lúdico no hospital: o olhar da criança. Journal of Human Growth and Development, v. 2, n. 24, p. 188-194, 2014.

FLICK, Uwe. Qualidade na pesquisa qualitativa. Porto Alegre: Artmed, 2009.

GIL, Antônio Carlos. Como elaborar projetos de pesquisa. 4. ed. São Paulo: Atlas, 2002.

GOMES-DA-SILVA, Pierre Normando. 0 jogo da cultura e a cultura do jogo: por uma semiótica da corporeidade. João Pessoa: Editora Universitária, 2011.

HOSTER, Paula Coimbra da Costa Pereira; ENUMO, Sônia Regina Fiorim; MOTTA LOSS, Alessandra Brumoro. Brincar e problemas de comportamento de crianças com câncer de classes hospitalares. Revista Psicologia: Teoria e Prática, v.16, n.1, p.127-140, 2014.

HUIZINGA, Johan. Homo Ludens. 8. ed. São Paulo: Perspectiva, 2014.

INSTITUTO NACIONAL DE CÂNCER. Coordenação de Prevenção e Vigilância. Particularidades do Câncer Infantil. In: ___. Estimativa 2008: Incidência de Câncer no Brasil. Disponível em: <http://www.inca.gov.br/conteudo view.asp?id=343>. Acesso em: 26 out.2016.

INSTITUTO NACIONAL DE CÂNCER. Estimativa 2016: Incidência de Câncer no Brasil. Rio de Janeiro, 2015.

INSTITUTO NACIONAL DE CÂNCER. Incidência, mortalidade e morbidade hospitalar por câncer em crianças, adolescentes e adultos jovens no Brasil: Informações dos registros de câncer e do sistema de mortalidade. Rio de Janeiro, 2016.

KOHLSDORF, Marina; SEIDL, Elaine Maria Fleury. Comunicação médico-cuidador-paciente em onco-hematologia pediátrica: perspectivas e desafios. Psicologia Argumento, v. 29, n. 66, p. 373-381, jul./set. 2011.

LE BRETON, David. As paixões ordinárias: antropologia das emoções. Petrópolis: Vozes, 2009.

LIMA, Pedro Augusto Leão; GOMES, Dinani. Jogos que auxiliam no tratamento de enfermidades: serious games. Revista Opara, v.4, n.1, p.57-70, 2014. 
MALTA, Julia Dias Santana; SCHALL, Virgínia Torres. Instrumento para a humanização do cuidado do câncer infanto-juvenil. Pediatria Moderna, v. 48, n.2, p.74-78, fev. 2012.

MENOSSI, Maria José; LIMA, Regina Aparecida Garcia. A problemática do sofrimento: percepção do adolescente com câncer. Revista da Escola de Enfermagem USP, v. 34, n. 1, p. 45-51, mar. 2000.

MITRE, Rosa Maria de Araújo; GOMES, Romeu. A promoção do brincar no contexto da hospitalização infantil como ação de saúde. Ciência \& Saúde Coletiva, v. 9, n.1, p. 147-154, 2004.

OATLEY, Keith; JENKINS, Jenniffer. Compreender as emoções. Lisboa: Instituto Piaget, 2002.

OLIVEIRA, Lecila Duarte Barbosa et al. A brinquedoteca hospitalar como fator de promoção no desenvolvimento infantil: relato de experiência. Revista Brasileira de Crescimento Desenvolvimento Humano, v.2, n.19, p. 306-312, 2009.

OLIVEIRA, Rosana Beatriz Gonçalves et al. Entrevista nas pesquisas qualitativas de enfermagem pediátrica. Revista Brasileira de Enfermagem, v.63, n.2, p. 300-306, 2010.

OLIVEIRA, Sâmela Soraya Gomes; DIAS, Maria da Graça; ROAZZI, Antônio. O lúdico e suas implicações nas estratégias de regulação das emoções em crianças hospitalizadas. Psicologia Reflexão e Crítica, v.1, n.16, p. 1-13, 2003.

PAULA, Ercília Maria Angeli Teixeira; FOLTRAN, Elenice Parise. Brinquedoteca Hospitalar: direito das crianças e adolescentes hospitalizados. Revista Conexão UEPG, v.3, n. 1, p. 20-23, 2007.

TAILLE, Yves De La; OLIVEIRA, Marta Kohl; DANTAS, Heloysa. Piaget, Vigotsk, Wallon: Teorias Psicogenéticas em discussão. 26. ed. São Paulo: Summus, 1992.

VAZ, Alexandre Fernandez; VIEIRA, Carmen Lúcia Nunes; GONÇALVES, Michelle Carreirão. Educação do corpo e seus limites: possibilidades para a Educação Física na classe hospitalar. Movimento, v. 1, n. 11, p.71-87, jan/abr. 2005. 


\section{Apoio:}

Agência de fomento: Fundação de Amparo a Ciência e Tecnologia de Pernambuco - FACEPE. Contribuição financeira para o material de coleta: FIAT ITALIANA. 